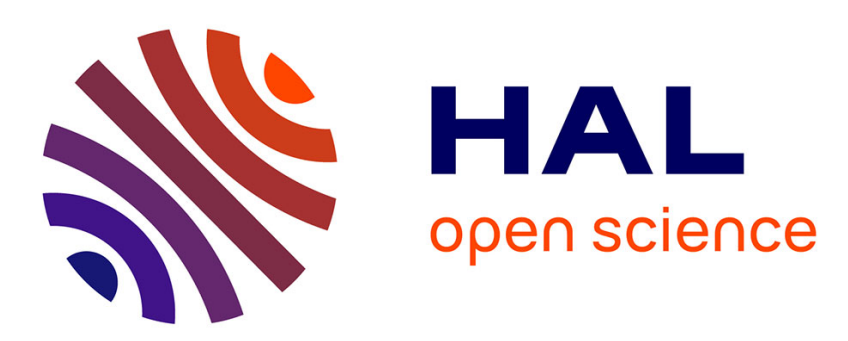

\title{
Standardization of antimicrobial testing of dental devices
}

Josette Camilleri, Teresa Arias Moliz, Ana Francisca Bettencourt, Joana

Costa, Filomena Martins, D Rabadijeva, Daniel Rodriguez, Livia Visai, Christèle Combes, Cher Farrugia, et al.

\section{- To cite this version:}

Josette Camilleri, Teresa Arias Moliz, Ana Francisca Bettencourt, Joana Costa, Filomena Martins, et al.. Standardization of antimicrobial testing of dental devices. Dental Materials, 2020, 36 (3), pp.59-73. 10.1016/j.dental.2019.12.006 . hal-03206165

\section{HAL Id: hal-03206165 \\ https://hal.science/hal-03206165}

Submitted on 23 Apr 2021

HAL is a multi-disciplinary open access archive for the deposit and dissemination of scientific research documents, whether they are published or not. The documents may come from teaching and research institutions in France or abroad, or from public or private research centers.
L'archive ouverte pluridisciplinaire HAL, est destinée au dépôt et à la diffusion de documents scientifiques de niveau recherche, publiés ou non, émanant des établissements d'enseignement et de recherche français ou étrangers, des laboratoires publics ou privés. 


\section{Open Archive Toulouse Archive Ouverte (OATAO)}

OATAO is an open access repository that collects the work of Toulouse researchers and makes it freely available over the web where possible

This is an author's version published in: http://oatao.univ-toulouse.fr/27617

Official URL: https://doi.org/10.1016/j.dental.2019.12.006

\section{To cite this version:}

Camilleri, Josette and Arias Moliz, Teresa and Bettencourt, Ana Francisca and Costa, Joana and Martins, Filomena and Rabadijeva, D and Rodriguez, Daniel and Visai, Livia and Combes, Christèle $\stackrel{5}{\mathrm{~S}}$ and Farrugia, Cher and Koidis, Petros T. and Neves, Cristina Bettencourt Standardization of antimicrobial testing of dental devices. (2020) Dental Materials, 36 (3). e59-e73. ISSN 0109-5641

Any correspondence concerning this service should be sent to the repository administrator: tech-oatao@listes-diff.inp-toulouse.fr 


\title{
Standardization of antimicrobial testing of dental devices
}

\author{
J. Camilleri ${ }^{a, k, 1}$, T. Arias Moliz ${ }^{b}$, A. Bettencourt ${ }^{c}$, J. Costa $^{d}$, F. Martins ${ }^{e}$,

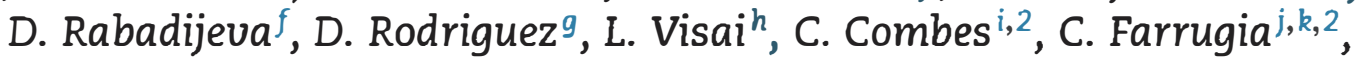 \\ P. Koidis ${ }^{1,2}$, C. Neves ${ }^{d, 2}$ \\ a School of Dentistry, Institute of Clinical Sciences, College of Medical and Dental Sciences, University of \\ Birmingham, Edgbaston, Birmingham, United Kingdom \\ b Department of Oral Microbiology, University of Granada, Granada, Spain \\ c Research Institute for Medicines (iMed.ULisboa), Faculty of Pharmacy, University of Lisbon, Lisbon, Portugal \\ ${ }^{`}$ Oral and Biomedical Sciences Research Unit (UICOB), Faculty of Dental Medicine, University of Lisbon, Lisbon, \\ Portugal \\ e Centro de Química e Bioquímica (CQB) and Centro de Química Estrutural (CQE), Department of Chemistry and \\ Biochemistry, Faculty of Sciences, University of Lisbon, Portugal \\ $\mathrm{f}$ Bulgarian Academy of Sciences, Institute of General and Inorganic, Bulgaria \\ $\mathrm{g}$ Technical University of Catalonia/Dept. Materials Science, Spain \\ h University of Pavia, Department of Molecular Medicine, Center for Health Technologies (CHT), UdR of INSTM, \\ Pavia, Italy \\ i CIRIMAT, Université de Toulouse, CNRS, Toulouse INP - ENSIACET, Toulouse, France \\ i School of Clinical Dentistry, Claremont Crescent, University of Sheffield, Sheffield, United Kingdom \\ k Department of Restorative Dentistry, Faculty of Dental Surgery, University of Malta, Malta \\ ${ }^{1}$ Department of Prosthodontics, School of Dentistry, Aristotle University of Thessaloniki, Greece
}

\section{A B S T R A C T}

Keywords:

Standardization

Antimicrobial testing

Dental devices

\begin{abstract}
Objective. Dental device is a very broad term that can be used to include any foreign material or product that is introduced in the host oral cavity to replace missing tissues. These devices are subjected to different environments which include dental hard tissues, tissue fluids, blood and saliva. All dental devices are continuously challenged microbiologically and a number of failures in clinical management are related to microbial colonization. Thus, the assessment of the antimicrobial properties of dental devices are extremely important. In this paper, a classification of dental devices is being proposed. This classification distinguishes the devices based on whether they are implantable or not, and also sub-classified based on their specific application and the substrate receiving the device.

Methods and Results. A literature search was conducted to identify how dental devices have been tested with relation to the microbial strains used and whether the testing has been performed in isolation or reported with other relevant tests such as material characterization and biological activity.
\end{abstract}

\footnotetext{
* Corresponding author at: School of Dentistry, Institute of Clinical Sciences, College of Medical and Dental Sciences, University of Birmingham, 5, Mill Pool Way, Edgbaston, Birmingham B5 7EG, United Kingdom.

E-mail address: J.Camilleri@bham.ac.uk (J. Camilleri).

${ }^{1}$ Main project lead

2 Section leads and equal contribution.

https://doi.org/10.1016/j.dental.2019.12.006
} 
The results of the literature review were analyzed and recommendations for antimicrobial testing of dental devices are proposed. These recommendations include the need for the setting up of pre-testing parameters such as ageing and the details of the pre-testing sterilization procedures, as these may affect the material chemistry and the specification for antimicrobial testing to be done with specific single strains or polymicrobial that are native to the region where the device is located are also suggested. Testing can be undertaken in vitro, ex vivo and in vivo. Since the antimicrobial and biological activities influence/condition one another and the material chemistry may affect both the antimicrobial and biological testing this document also makes recommendations regarding biological assessment which can be carried out in isolation or integrated with the microbiological testing and also material testing methods including chemical and physical characterization of bulk, surface, eluted and degraded materials as well as physical characterization methods.

Significance. The level of standardization of antimicrobial testing for the dental devices needs to be based on the device location and host interaction in order to increase the clinical applicability of the mentioned tests.

(c) 2019 The Academy of Dental Materials. Published by Elsevier Inc. All rights reserved.

\section{Contents}





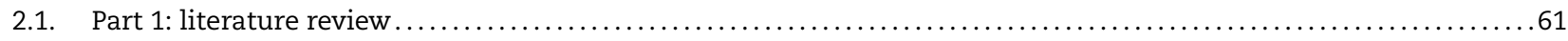

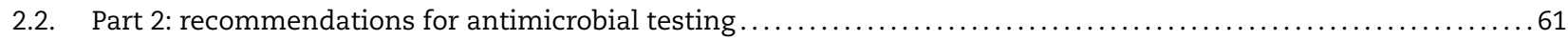



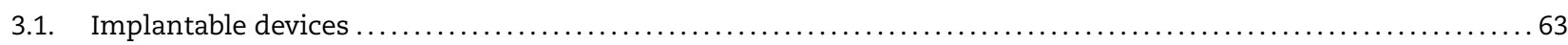

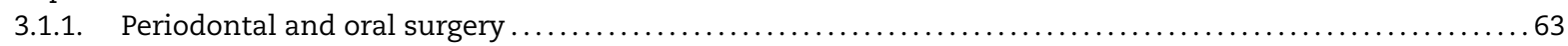

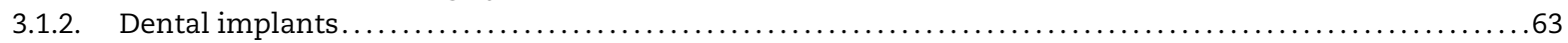

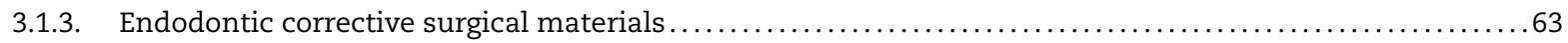

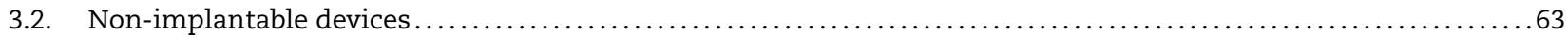

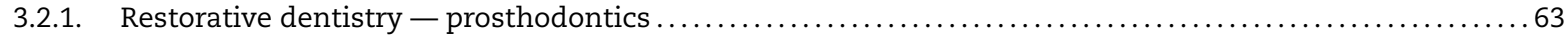

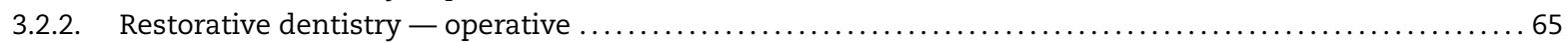



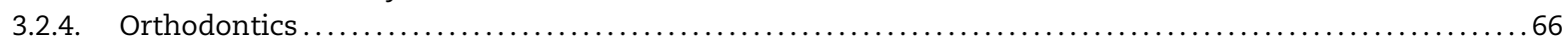

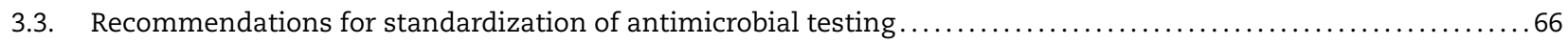

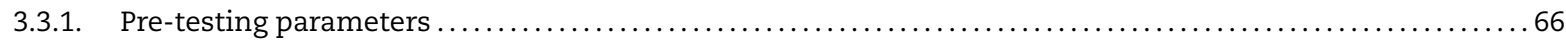

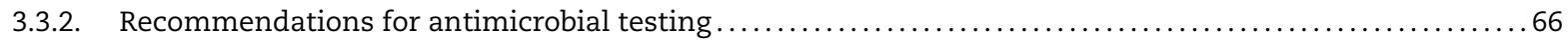

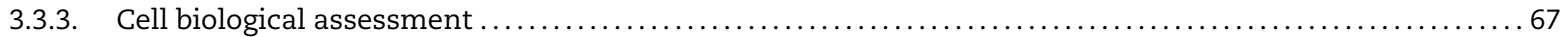

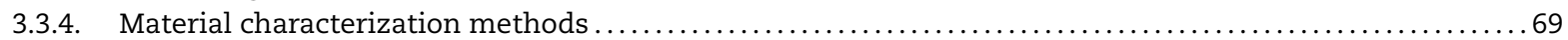

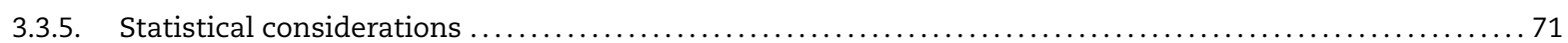

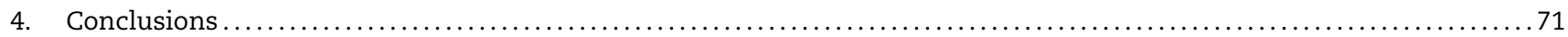

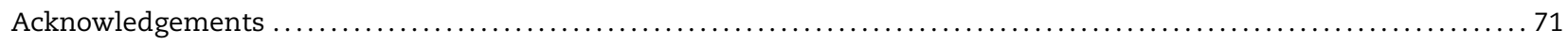

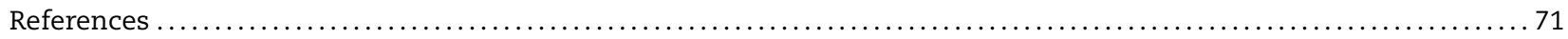

\section{Introduction}

There is no proposed definition of a dental device. Dental devices are part of the medical devices which are defined as any instrument, apparatus, appliance, software, material, or other article used in medical practice. This includes devices used alone or in combination with software necessary for diagnosis, prevention, monitoring, treatment or alleviation of disease [1]. A dental device can thus also be similarly defined and includes the specific use in the oral cavity. A dental device is any foreign material or product that is introduced in the host oral cavity to replace missing tissues. It is a very broad term that encompasses several devices used in the oral cavity, which serve different purposes, interact with different tissues and are in contact with different oral environments. The oral cavity has various commensal microorganisms that are not pathogenic to the host. Thus, the desired level of antimicrobial activity of dental devices varies with the location, clinical use of the device and the environment in which it is placed and the time of implantation.

Various methods for testing antimicrobial activity of dental devices are described in different papers testing both established materials already in clinical use and prototypes that 


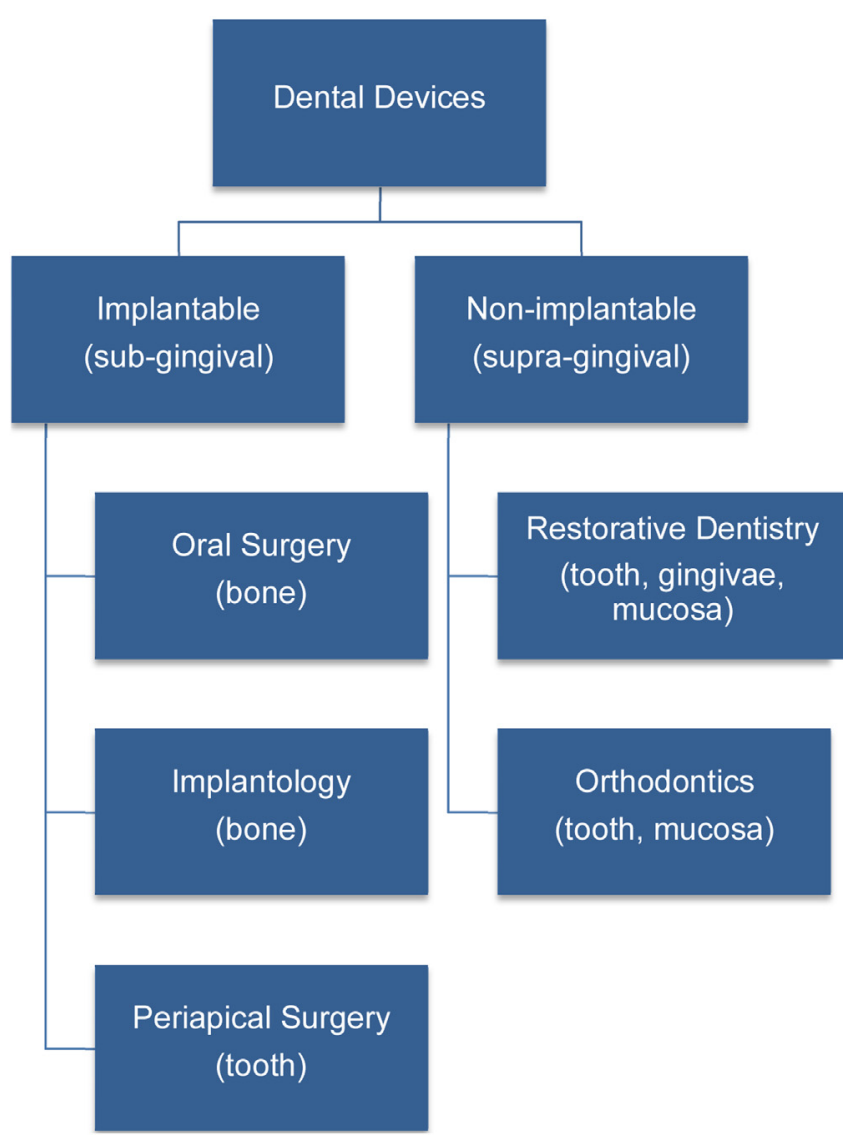

Fig. 1 - Schematic representation of the classification of devices for use in dentistry taking into consideration the specific use and substrate where the device is placed.

still need to be further evaluated. Due to the wide range of dental devices, it is necessary to classify and consider the clinical needs prior to assessing the antimicrobial activity. Furthermore, the level of testing should reflect the degree of risk posed on the host. Dental devices can also be classified differently if they have anti-microbiological effects either as a prevention device of future infections or as a treatment device used on already installed infection. Testing should enclose different microbiological characteristics of these different environments. A basic classification is proposed for dental devices as shown in Fig. 1. This classification distinguishes the devices on whether they are implantable or not, and sub-classified depending on their specific application and the substrate receiving the device.

In dentistry, failure in clinical management is mostly related to bacterial infection. Thus, all dental devices should be tested for antimicrobial properties. Unlike the testing of biological properties, there are no specific standards for the testing of antimicrobial properties.

The specific objectives of this paper are to (i) review the literature to identify how dental devices have been tested with relation to the microbial strains used and whether the testing has been performed in isolation or reported with other relevant tests such as material characterization and biological activity; (ii) suggest a level of standardization of antimicrobial testing for the dental devices based on the device location and host interaction.

\section{Methodology}

\subsection{Part 1: literature review}

Based on the classification proposed in Fig. 1, where dental devices were classified as implantable and non-implantable and sub-classified on the field of specialization and the contact with specific environments, searches were conducted using PubMed search engine for devices subdivided into 7 groups namely:

- Implantable for oral surgery

- Implantable for implantology

- Implantable for periradicular surgery

- Non-implantable for restorative dentistry-prosthodontics

- Non-implantable for restorative dentistry-operative dentistry

- Non-implantable for restorative dentistry-endodontics

- Non-implantable for orthodontics

For all the groups the following keywords were included: antimicrobial, antibacterial, dental/oral. Following this preliminary search, other terms that were more specific to each group were inserted to reduce the number of articles retrieved. Articles within a 10-year range were included i.e., from 2008 to 2018. Articles not written in English, all review articles and articles where antimicrobials were added to the materials but the testing of other properties was undertaken were excluded. The key words and outputs are shown in Table 1.

For the oral surgery all the five searches indicated in Table 1 were undertaken. For the Implantology group, the output of 695 articles was further filtered to select the most relevant papers: articles with materials tested without specifying a dental application, use of bacterial strains not specific to oral applications, biomaterials not used to manufacture dental implants, or coatings for dental implants, materials/pieces not used for implantation in bone or to test/report medical treatment associated to dental implants or cases in which the antimicrobial testing was not significant, were excluded. These exclusion criteria led to the selection of 68 articles.

For the periradicular surgery the introduction of the term root-end reduced the search to 17 articles and six fulfilled the criteria. For the non-implantable materials, the introduction of specific key words also reduced the number of articles retrieved and all these were read and the exclusion criteria applied as indicated in Table 1.

The details per output included the author's first name, date of publication, full citation, the materials tested, the strains used, whether the testing was performed in vitro, ex vivo or in vivo and also details of any other testing performed.

\subsection{Part 2: recommendations for antimicrobial testing}

Based on the results of the literature review, recommendations for antimicrobial testing of dental devices are given. These recommendations will take into consideration pre-testing 
Table 1 - Summary of keywords searched for each topic and number of outputs.
Classification of device

\begin{tabular}{|c|c|c|c|c|}
\hline Classification of device & & Key words & Articles & After exclusion \\
\hline \multirow{17}{*}{ Implantable } & \multirow{6}{*}{ Oral surgery } & Antimicrobial, OR Antibacterial, AND Dental, OR Oral & 1038156 & Not searched \\
\hline & & Antimicrobial, OR Antibacterial, AND Periodontal surgery materials & 106 & 9 \\
\hline & & Antimicrobial, OR Antibacterial, AND Oral graft materials & 55 & 1 \\
\hline & & Antimicrobial, OR Antibacterial, AND Oral guided tissue regeneration membrane & 91 & 4 \\
\hline & & Antimicrobial, OR Antibacterial, AND Periodontal surgery gel & 27 & 0 \\
\hline & & Antimicrobial, OR Antibacterial, AND Oral surgery gel & 55 & 0 \\
\hline & Implantology & Antibacterial OR antimicrobial AND (Dental OR Oral) AND Implant NOT Review & 695 & 68 \\
\hline & \multirow{2}{*}{$\begin{array}{l}\text { Peri-radicular } \\
\text { surgery }\end{array}$} & Antimicrobial, OR Antibacterial, AND Endodontics & 1757 & Not searched \\
\hline & & Antimicrobial, OR Antibacterial, AND Endodontics AND Root-end & 17 & 6 \\
\hline & \multirow{5}{*}{$\begin{array}{l}\text { Restorative } \\
\text { Dentistry- } \\
\text { Prosthodontics }\end{array}$} & Antimicrobial OR Antibacterial AND (Dental OR Oral) AND Prosthodontics & 1738 & Not searched \\
\hline & & Antimicrobial, OR Antibacterial, AND (Dental OR Oral) AND Prosthodontics AND Acrylic Dentures & 124 & 49 \\
\hline & & Antimicrobial OR Antibacterial, AND (Dental OR Oral) AND prosthodontics AND Ceramics & 79 & 3 \\
\hline & & Antimicrobial OR Antibacterial, AND (Dental OR Oral) AND Metal Crowns & 66 & 1 \\
\hline & & Antimicrobial, OR Antibacterial, AND Dental Amalgam & 64 & 7 \\
\hline & Restorative & Antimicrobial, OR Antibacterial, AND Dental Composite & 59 & 24 \\
\hline & \multirow{4}{*}{ Dentistry-Operative } & Antimicrobial, OR Antibacterial, AND Dental Glass Ionomer & 329 & 93 \\
\hline & & Antimicrobial, OR Antibacterial, AND Luting & 132 & 11 \\
\hline \multirow[t]{9}{*}{ Non-implantable } & & Antimicrobial, OR Antibacterial, AND Endodontics & 1757 & Not searched \\
\hline & & Antimicrobial, OR Antibacterial, AND Endodontics, AND Pulp Capping Materials & 46 & 9 \\
\hline & Restorative & Antimicrobial, OR Antibacterial, AND Endodontics, AND Endodontic Filling Materials & 98 & 40 \\
\hline & Dentistry- & Antimicrobial, OR Antibacterial, AND Endodontics, AND Gutta-percha & 63 & 13 \\
\hline & \multirow[t]{3}{*}{ Endodontics } & Antimicrobial, OR Antibacterial, AND Endodontics, AND Resilon & 12 & 2 \\
\hline & & Antimicrobial, OR Antibacterial, AND Endodontics, AND Sealers & 91 & 63 \\
\hline & & Antimicrobial, OR Antibacterial, AND Endodontics, AND Posts & 2 & 0 \\
\hline & \multirow{2}{*}{ Orthodontics } & Antimicrobial, OR Antibacterial, AND (Dental OR Oral) AND Orthodontics & 635 & Not searched \\
\hline & & Antimicrobial, OR Antibacterial, AND (Dental OR Oral) AND Orthodontics AND Brackets AND wires & 177 & 61 \\
\hline
\end{tabular}


parameters such as ageing and the method of pre-sterilization of the device that needs to be noted in every test. Furthermore, the selection of the strain for both mono-species and multispecies testing and recommendations for the microbiological testing of the devices are made, and include in vitro, ex vivo and in vivo testing. Suggestions for integrated microbial and cellular testing and the need of material characterization including chemical characterization methods for bulk, surface, elution and degraded material and physical and mechanical characterization are also made.

\section{Results}

After the inclusion and exclusion criteria were applied the articles to be included for each subgroup are shown in Table 2.

\subsection{Implantable devices}

\subsubsection{Periodontal and oral surgery}

In the periodontal and oral surgery materials, after excluding implants, only 14 papers were selected and a further 2 were removed due to hits in more than one search term for the same paper. A total of 12 papers from the last 10 years, were therefore reviewed. Most papers investigated materials developed for use as guided tissue regeneration (GTR) or guided bone regeneration (GBR) membranes (10 papers) while only one of the reviewed papers investigated a scaffold and one investigated microspheres. None of the reviewed articles studied other types of grafts, gels or devices used in oral and periodontal surgery. In vitro methods are the most common method of antimicrobial testing, with variations of the agar diffusion test were the most commonly used antimicrobial test ( 6 publications) and most of the studies (8 publications) only tested for antimicrobial properties using one antimicrobial test. None of the reviewed literature used ex vivo methods and only one study used an additional in vivo test on Beagle dogs as an animal model. Nonetheless, this model was only used to assess histopathological tissue changes and not antimicrobial efficacy. Fusobacterium nucleatum and Streptococcus mutans were the most common microorganisms used for antimicrobial testing (4 publications each) and all testing was done using single species infections. Although 7 different publications tested antimicrobial properties against multiple organisms, only one publication used 2 strains of the same microorganism.

Eight out of the 12 reviewed studies included material characterization, as well as biological testing in addition to the antimicrobial testing. And out of all of the reviewed literature only 2 articles used or referred to the commercially available equivalent in their publication. Lack of standardization in antimicrobial testing of materials used in oral and periodontal surgery was observed with a large variation of organisms being tested and in one case the strains used were not even described. There is mostly disparity when testing for bone regenerative materials as some studies only test for typical osteomyelitis causing organisms such as Staphylococcus aureus, but do not include other typical oral microorganisms in their testing.

\subsubsection{Dental implants}

For the implantable devices, among the 68 papers related to the dental implants sub-group, 52 reported antimicrobial studies involving bacteria single species testing and 16 used bacteria multi-species together for the testing. Porphyromonas gingivalis (24 papers), Streptococcus mutans (19 papers), Streptococcus sanguinis (15 papers) and Streptococcus gordonii (10 papers) were the most used bacteria strains. All the 16 studies implementing more than one bacterial strain in the testing investigated biofilm formation. 55 papers reported in vitro microbiological testing, three of which also presented an in vivo study. One paper was dedicated only to in vivo assessment. The in vitro methods were dominated by colony forming unit (CFU) counting, followed by minimum inhibitory concentration (MIC) and MTT and live/dead. Twenty-seven of the papers included other biological assessment and 32 papers included characterization of the materials. Commercially pure titanium was by far the most common material tested (54 papers). Titanium alloy (Ti6Al4V, 4 papers), and hydroxyapatite, PEEK, zirconia, and PLGA were almost single instance materials (one or two papers).

\subsubsection{Endodontic corrective surgical materials}

The root-end filling materials were only investigated for antimicrobial characteristics in 6 papers in the last 10 years. The papers investigated specifically hydraulic calcium silicate cements including mineral trioxide aggregate (MTA) and Biodentine and some antimicrobial additives such as silver nanoparticles and hinokitiol. In 5 papers in vitro methods including agar diffusion and the microdilution broth methods were used and only 1 paper used dentine substrate and conducted intratubular infection method thus using an ex vivo method. Enterococcus faecalis was the most frequent tested microorganism (6 papers); either as the only microorganism employed (4 papers) or with other oral bacteria. Two of the papers used more than one bacterial strain but only 1 used a polymicrobial culture. Only 1 paper carried out material characterization and biological testing of the materials.

\subsection{Non-implantable devices}

\subsubsection{Restorative dentistry - prosthodontics}

In the group of prosthetic restorative materials, after search specifications were applied, only 4 articles were found that studied antimicrobial characteristics in the fixed prosthetic field - one in metal crown, and 3 in ceramics. The antimicrobial properties of surface treatments of each material, such as plasma, silver nanoparticles coating and polarization-induced treatments were studied. These in vitro studies used direct contact and CFU counting. Streptococcus mutans was used in all papers, either as the only tested microorganism (2 papers) or with other oral bacteria. Two papers that described new surface treatments also carried out a variety of structural and adhesion studies and biological testing.

On the contrary, in the removable prosthetic field, a considerable amount of work in the antimicrobial testing of acrylic dentures - 49 articles were found. So, this group was further subdivided into three sub-groups: drug loaded acrylic resins, drug exposure acrylic resins (e.g. testing after disinfection) and drug coated acrylic resins (e.g. denture adhesives). 


\begin{tabular}{|c|c|c|c|c|c|c|c|c|c|c|}
\hline \multirow[t]{2}{*}{ Device location } & \multirow[t]{2}{*}{ Specialization } & \multirow[t]{2}{*}{ Device tested } & \multirow{2}{*}{$\begin{array}{l}\text { Articles included } \\
\text { Single }\end{array}$} & \multicolumn{2}{|c|}{ Species } & \multicolumn{3}{|c|}{ Testing performed } & \multicolumn{2}{|l|}{ Other tests } \\
\hline & & & & Multi $^{\mathrm{a}}$ & In vitro & Ex vivo & In vivo & Characterization & Biological testing & \\
\hline \multirow{6}{*}{ Implantable } & \multirow{5}{*}{ Oral Surgery } & Oral GTR and GBR membranes & 10 & 10 & 0 & 10 & 0 & 1 & 6 & 6 \\
\hline & & Oral grafts & 2 & 2 & 0 & 2 & 0 & 0 & 2 & 2 \\
\hline & & Oral and periodontal gels & 0 & 0 & 0 & 0 & 0 & 0 & 0 & 0 \\
\hline & & Dental implants & 68 & 52 & 16 & 55 & 10 & 3 & 32 & 16 \\
\hline & & Root-end & 6 & 5 & 1 & 5 & 1 & 0 & 1 & 1 \\
\hline & \multirow{3}{*}{ Prosthodontics } & Acrylic dentures & 49 & 44 & 5 & 48 & 0 & 1 & 15 & 6 \\
\hline \multirow{13}{*}{ Non-implantable } & & Ceramics & 3 & 2 & 1 & 3 & 0 & 0 & 2 & 2 \\
\hline & & Metal crowns & 1 & 1 & 0 & 1 & 0 & 0 & 0 & 0 \\
\hline & \multirow{5}{*}{ Operative dentistry } & Dental amalgam & 7 & 6 & 1 & 4 & 2 & 1 & 0 & 2 \\
\hline & & Dental composite resin & 24 & 18 & 6 & 23 & 1 & 0 & 0 & 4 \\
\hline & & Dental glass ionomer & 93 & 91 & 2 & 90 & 2 & 13 & 0 & 0 \\
\hline & & Luting cements & 11 & 11 & 0 & 9 & 1 & 1 & 1 & 1 \\
\hline & & Pulp preservation & 9 & 8 & 1 & 8 & 0 & 1 & 0 & 2 \\
\hline & \multirow{5}{*}{ Endodontics } & Gutta-percha & 13 & 11 & 2 & 5 & 8 & 0 & 0 & 0 \\
\hline & & Resilon & 2 & 2 & 0 & 0 & 2 & 0 & 0 & 0 \\
\hline & & Sealers & 55 & 53 & 2 & 46 & 12 & 0 & 3 & 3 \\
\hline & & Experimental sealers & 8 & 7 & 1 & 8 & 0 & 0 & 2 & 3 \\
\hline & & Posts & 0 & 0 & 0 & 0 & 0 & 0 & 0 & 0 \\
\hline & Orthodontics & Brackets/wires & 61 & 54 & 7 & 46 & 2 & 14 & 36 & 8 \\
\hline
\end{tabular}


Most of these studies tested for Candida species - mostly Candida albicans, which was referred to as the primary cause of denture-related stomatitis. Single Candida albicans was tested in 28 studies, 7 cultured from clinical samples. Streptococcus mutans strains were the more relevant bacteria for antimicrobial tests (10 studies). Two studies tested only for Staphylococcus aureus and Pseudomonas aeruginosa, both focusing on bacteria that can form denture biofilm and can cause other systemic diseases like respiratory pneumonia. Five studies tested total clinical biofilm and one was a randomized clinical study.

Most of the in vitro testing consisted of only one method, usually direct contact assays (e.g. CFU counting by agar plate culture method) and biofilm evaluation for bioactivity (dead/live staining - XTT reduction), biomass (crystal violet assay) and scanning electron microscopy (SEM). Nevertheless, at least 19 papers used two or three combined methods.

A considerable amount of studies of drug loaded acrylic resins also included material characterization and biological studies. Two studies of drug exposure or drug coated acrylic resins also included biological or material characterization evaluation.

\subsubsection{Restorative dentistry - operative}

In the group of restorative dentistry and operative materials/devices the initial search revealed also a great number of articles. In the sub-group for amalgam 64 were identified, for composite resin 59, for glass ionomer 329, while for luting cements 132. After the application of inclusion and exclusion criteria, the secondary outcomes limited the articles to a total number of 135. More specifically, 7 articles for amalgam, 24 for composite resin, 93 for glass ionomer and 11 for luting cements. From the total 135 studies, 15 were in vivo, 6 ex vivo while 126 were in vitro or in combination with the rest. In 104 of the 135 papers Streptococcus mutans was the strain tested, primarily as single and in a significant $\%$ of papers exclusively (52/135). Characteristically, in the 93 papers for glass ionomer cements 4 papers did not include $S$. mutans but instead other species (L. casei (n 2), A. viscosus, C. albicans, S. milleri and $E$. faecalis). In one study 11 species were involved (S. mutans, S. salivarius, S. sorbinus, A. viscosus, A. naeslundii, A. odontolyticus, L. acidophilus, L. caesi, P. gingivalis, Prevotella intermedia, A. actinomycetemcomitans), one study used 6 strains, three studies 5 while four studies 4 strains. Similarly, in the reviewed 24 papers for composite resins $S$. mutans was used in 16 (in 10/16 exclusively), in one study 6 different strains (P. gingivalis, P. intermedia, P. nigrescens, A. actinomycetemcomitans, F. nucleatum and E. faecalis), in one study 4 (P. gingivalis, P. intermedia, A. actinomycetemcomitans and F. nucleatum), while in 4 studies a dental plaque microcosm biofilm model was used.

For the in vitro testing, the methods usually applied were direct contact assays (e.g. CFU counting), ADT, BAT, ABCT and biofilm evaluation for bioactivity (dead/live staining - XTT reduction), biomass (cristal violet assay), adhesion assays, MTT (in 10 studies) or MTS, fluorescence or confocal imaging and SEM. NMR was used in one study and very limited ELISA and rtPCR (in 4 studies). Ageing procedures were applied in 7 studies and thermocycling in only 1.

\subsubsection{Restorative dentistry - endodontics}

For the non-implantable devices, sub-groups restorative dentistry and endodontics after the inclusion and exclusion criteria were applied, the quantity of papers reduced significantly. Only 9 papers tested antimicrobial characteristics of pulp capping materials. From the 9 papers, 5 used the agar diffusion test to assess the antimicrobial activity; one evaluated the leachate rather than the materials themselves. The rest of the in vitro studies used the MIC, direct contact test and CFU counting. In general, the materials were tested using a variety of oral bacteria and bacteria related to dental caries. One paper used E. faecalis which is more linked to failed root canal therapy. Two of the papers included biological testing. None of the papers characterized the materials used. One of the papers was a clinical study isolating bacteria in samples of carious dentine in vivo. This was the only study that used a multispecies biofilm.

Inside the root canal, searches for endodontic filling materials yielded 40 articles that fell within the search specifications. Further searches for sealers and gutta-percha, resulted in finding more related papers. There were 13 papers published on antimicrobial activity of gutta-percha. Nine papers out of the total 13 that met the criteria used single species of $E$. faecalis for testing, 2 used more than 1 species but tested separately and only 2 used multispecies biofilm. Dentine substrate was used in 8 of the 13 papers. Although gutta-percha was tested in these studies, it was used either as control to test the efficacy of sealers used or else modified versions were investigated rather than the gutta-percha per se. Thus, although this material is used extensively in clinical endodontics, it is not tested very rigorously. For Resilon, another core filling material, only 2 papers met the criteria. Both papers used a single species of $E$. faecalis and dentine as a substrate was used in the antimicrobial testing. All the papers reviewed did not include any material characterization or any biological testing.

A considerable amount of work has been published in the antimicrobial testing of root canal sealers. Several prototype sealers have also been tried. So, this group was further subdivided into two, clinically available sealers and experimental sealers. This was done to be able to differentiate the testing undertaken for the clinically available materials and the experimental ones. For the testing of the clinically available sealers, 53 out of 55 used a single species culture. Thirty four of the papers tested only one strain and 21 included other microorganisms associated with the endodontic infections and also Candida albicans. Most of the in vitro testing consisted of only one method. Three papers used both in vitro and ex vivo methods and 11 used only ex vivo methods that included dentine substrate. There were no in vivo studies. Three papers included material characterization and 3 biological testing.

For the experimental sealers, seven out of the eight papers used E. faecalis single microorganism for testing. All the methods employed were in vitro without using dentine substrate. Two studies included material characterization and 3 biological assessment of the materials. No literature on the antimicrobial properties of post materials has been found. 
Table 3 - Specific strains suggested for first line device testing depending on location and procedure performed.

\begin{tabular}{|c|c|c|c|c|}
\hline Device & Procedure & Substrate & Strain & ATCC number \\
\hline \multirow{3}{*}{ Implantable } & Oral Surgery & Bone & S. aureus/P. gingivalis & $25923 / 33277$ \\
\hline & Implantology & Bone & P. gingivalis & 33277 \\
\hline & Periapical Surgery & Tooth (Dentin) & E. faecalis & 29212 \\
\hline \multirow{6}{*}{$\begin{array}{l}\text { Non- } \\
\text { Implantable }\end{array}$} & \multirow{4}{*}{ Restorative Dentistry } & Oral Mucosa & S. oralis & 10557 \\
\hline & & Tooth (Enamel) & S. mutans & 25175 \\
\hline & & Tooth (Dentin-Crown) & S. mutans & 25175 \\
\hline & & Tooth (Dentin-Root) & E. faecalis & 29212 \\
\hline & \multirow{2}{*}{ Orthodontics } & Oral Mucosa & S. oralis & 10557 \\
\hline & & Tooth (Enamel) & S. mutans & 25175 \\
\hline
\end{tabular}

\subsubsection{Orthodontics}

A significant amount of work has been published in the antimicrobial testing of materials for orthodontic application, divided in two major fields: drug-loaded orthodontic cement systems (e.g. resin-based or glass ionomer cements) and nanoparticles coated brackets/wires (e.g. silver). For the evaluation, 54 of the papers used a single species with all of them except one opting for the strain Streptococcus mutans. The remaining papers included other microorganisms associated with the oral cavity (e.g.Streptcoccus sanguinis, Porphyromonas gingivalis) and also Candida albicans. Multi-species testing was performed in 6 papers which used human saliva as the inoculum resembling the microcosm oral biofilm environment. Additionally, in 1 paper, the presence of 16 g-negative periodontal-related microorganisms were tested on metallic brackets. The in vitro testing consisted mainly of direct contact assays (e.g. agar diffusion assay) and biofilm evaluation (e.g. dead/live staining, SEM). Two papers evaluating orthodontic brackets used ex vivo methods that included extracted maxillary premolars. There were 14 in vivo studies with all of them with humans except one using rats. Thirty-six papers included material characterization and 8 cytotoxicity studies.

\subsection{Recommendations for standardization of antimicrobial testing}

Based on the results of the literature review, it is clear that there is a lack of standardization of testing at all levels. Furthermore, data such as ageing and pre-sterilization if any is not always included. Most of the studies in each field of research did not include material characterization nor cell biological assessment to evaluate the interplay between the antimicrobial efficacy and damage to the host. The following recommendations are thus being proposed to alleviate this lack of standardization.

\subsubsection{Pre-testing parameters}

The following parameters need to be noted when undertaking antimicrobial testing of dental devices. These include the ageing of the specimens as well as the pre-sterilization method used, if any.

3.3.1.1. Ageing. Ageing of materials and devices refers to the gradual process in which the properties of a material, structure, or system, change (for better or worse), over time or with use, due to biological, chemical, mechanical or physical agents. Ageing affects the shelf life of a device and changes the device profile thus altering its characteristics. Artificial age- ing may be necessary for a number of tests. If the material or device is artificially aged this must be done following standard methods. The accelerated ageing methods and the specific duration of the test should also be noted. For all devices, cycles of acidic/neutral/basic $\mathrm{pH}$ for a given time are necessary. In addition:

- For implantable devices incubation in simulated body fluid at $37 \pm 1^{\circ} \mathrm{C}$ is recommended;

- For non-implantable devices the dynamic ageing dependent on the specific application is recommended. This should follow specifications listed in ISO/TS 11405:2015 [2].

3.3.1.2. Pre-sterilization. Pre-sterilization of the device is generally necessary prior to antimicrobial testing. Presterilization should be performed following established standard protocols outlined in ISO standards and should be in line with the device use in the clinical setting. Importantly, the methods employed neither shall affect the material properties nor the antimicrobial properties. The ISO standards outline the use of ethylene oxide in ISO 11135-1:2007 [3], radiation in ISO 11137-1:2015 [4], moist heat in ISO 17665-1:2006 [5], dry heat in ISO 20857:2010 [6], low temperature sterilization and formaldehyde in ISO 25424:2009 [7] and liquid chemicals in ISO 14160:2011 [8]. It is important that all microorganisms are eliminated and tests should be conducted to ensure a microorganism free device prior to the start of testing as indicated in the relevant ISO standards $[9,10]$.

\subsubsection{Recommendations for antimicrobial testing}

3.3.2.1. Description of the relevant strains. The testing can be performed using single or multiple microbial strains. The most relevant strains to be used for testing, categorized according to the material classification in Fig. 1 are listed in Table 3. In each case there is only one strain suggested for use as first line material testing. The strains are chosen as they are native to the location where the material is being implanted. Since most of the oral infections are polymicrobial, it is also important to test the antimicrobial activity of the materials against multi-microbial cultures (Table 4 ) as second line testing procedure. Non-implantable devices in contact with the oral mucosa should also be tested for the relevant strains of fungi, like Candida albicans using ATCC 10231 strain.

3.3.2.2. Microbiological testing. For microbiological assessment of devices used for dental applications, two sets of controls are necessary. A positive and a negative control are needed for the claim made and for each experimental tech- 
Table 4 - Specific strains suggested for multi species microbial testing depending on location and procedure performed.

\begin{tabular}{|c|c|c|c|c|}
\hline Device & Procedure & Substrate & Strain & ATCC number \\
\hline \multirow{3}{*}{ Implantable } & \multirow[t]{3}{*}{ Oral Surgery } & \multirow[t]{3}{*}{ Bone } & P. gingivalis, & 33277 \\
\hline & & & F. nucleatum, & 25586 \\
\hline & & & P. intermedia & 25611 \\
\hline \multirow{24}{*}{$\begin{array}{l}\text { Non- } \\
\text { Implantable }\end{array}$} & \multirow[t]{3}{*}{ Implantology } & \multirow[t]{3}{*}{ Bone } & P. gingivalis, & 33277 \\
\hline & & & F. nucleatum, & 25586 \\
\hline & & & P. intermedia & 25611 \\
\hline & \multirow[t]{4}{*}{ Periapical Surgery } & \multirow[t]{3}{*}{ Tooth (Dentin) } & E. faecalis, & 29212 \\
\hline & & & F. nucleatum, & 25586 \\
\hline & & & A. naeslundii & 12104 \\
\hline & & \multirow[t]{3}{*}{ Oral Mucosa } & S. oralis, & 10557 \\
\hline & \multirow{11}{*}{$\begin{array}{l}\text { Restorative } \\
\text { Dentistry }\end{array}$} & & S. gordonii, & 10558 \\
\hline & & & S. sanguinis & 10556 \\
\hline & & \multirow[t]{3}{*}{ Tooth (Enamel) } & S. mutans, & 25175 \\
\hline & & & S. sobrinus, & 33399 \\
\hline & & & A. naeslundii & 12104 \\
\hline & & \multirow[t]{3}{*}{ Tooth (Dentin-Crown) } & S. mutans, & 25175 \\
\hline & & & S. sobrinus, & 33399 \\
\hline & & & A. naeslundii & 12104 \\
\hline & & \multirow[t]{3}{*}{ Tooth (Dentin-Root) } & E. faecalis, & 29212 \\
\hline & & & F. nucleatum, & 25586 \\
\hline & & & A. naeslundii & 12104 \\
\hline & \multirow{6}{*}{ Orthodontics } & \multirow[t]{3}{*}{ Oral Mucosa } & S. oralis, & 10557 \\
\hline & & & S. gordonii, & 10558 \\
\hline & & & S. sanguinis & 10556 \\
\hline & & \multirow[t]{3}{*}{ Tooth (Enamel) } & S. mutans, & 25175 \\
\hline & & & S. sobrinus, & 33399 \\
\hline & & & A. naeslundii & 12104 \\
\hline
\end{tabular}

nique undertaken. A positive control is any well-characterized material and/or substance that, when evaluated by a specific test method, demonstrates the suitability of the test system to yield a reproducible, appropriately positive or reactive response in the test system. For microbiological assessment, the positive control is an anti-infective material demonstrating X-log reduction in biofilm cells and planktonic bacteria. A negative control is any well-characterized material and/or substance that, when evaluated by a specific test method, demonstrates the suitability of the test system to yield a reproducible, appropriately negative, non-reactive or minimal response in the test system. The negative control can be used to define background values. For microbiological assessment, a material allowing maximal biofilm formation is suggested.

A specific threshold is not necessary for dental devices. However, one should take into consideration the conflicting properties of antimicrobial activity and the cytotoxicity caused by the device when in clinical use. Various levels of testing need to be undertaken including in vitro, ex vivo and in vivo testing.

For in vitro testing, at least one method from each sub-type should be employed as each method has its limitations. If only antimicrobial activity testing is being undertaken, then at least two methods from this sub-type should be used (Table 5). For tests listed in Table 5, the following should be noted:

- Time span

- Time of contact

Refer to Table 6 for specific details of tests.

For ex vivo testing (testing performed outside the living organism but using substrates obtained from the living organism before or after placement of a device) one basic methodology is the intra-tubular infection test for devices in contact with infected dentin. The same methodologies as those employed for in vitro testing, i.e., antimicrobial activity testing (Table 5), are to be used for ex vivo testing but including the related substrate in the assessment. At least two methods of assessment should be considered. The testing can be performed with the device placed in a living organism and retrieved for testing or the substrate can be extracted from the living organism prior to carrying out the test.

In vivo testing is all the testing that is performed clinically. Testing should be undertaken in the living organism. Such tests include in vivo imaging techniques in animal models, radiographic and/or computer tomography techniques and other clinical methods depending on the site being tested. The list of tests and their suitability depending on specific parameters are shown in Table 6.

\subsubsection{Cell biological assessment}

Elimination of microbial strains from a dental device can make this device cyto/geno-toxic. Thus, cell biological assessment should be carried out following recommendations set out in ISO 7405: 2008 [11] and ISO 10993-5: 2009 [12]. The testing should be conducted on specific cell lines depending on the site of implantation of the device. Primary cells cultivated from living organism may be used for further testing. The suggested cell lines, depending on the site of implantation, are reported in Table 7. Three levels of testing are suggested including static tests, proliferation migration assays and genotoxicity testing. Finally integrated microbial and cellular testing should be also undertaken. 
Table 5 - In vitro testing methods for testing dental devices.

\begin{tabular}{|c|c|c|}
\hline Testing type & Testing methodology & Observations \\
\hline Basic tests & $\begin{array}{l}\text { Agar diffusion, } \\
\text { Direct contact test, } \\
\text { Minimum inhibitory concentration (MIC), } \\
\text { Minimum bactericidal/fungicidal } \\
\text { concentration (MBC/MFC), } \\
\text { Biofilm inhibitory concentration (BIC) }\end{array}$ & $\begin{array}{l}\text { For biofilm inhibitory concentration (BIC), } \\
\text { the remaining biofilm after } \\
\text { drug-treatment needs to be vortexed } \\
\text { before evaluation }\end{array}$ \\
\hline Antimicrobial activity testing & $\begin{array}{l}\text { Colony forming unit counting, } \\
\text { Confocal laser scanning microscopy } \\
\text { (CLSM), } \\
\text { Enzymatic activity, } \\
\text { Fluorescence in situ hybridization (FISH), } \\
\text { Nucleic acid techniques e.g. Polymerase } \\
\text { chain reaction (PCR) }\end{array}$ & $\begin{array}{l}\text { The antimicrobial activity tests need to be } \\
\text { performed either for direct or indirect } \\
\text { contact. For the indirect contact, the } \\
\text { materials releasing antimicrobials need } \\
\text { to be previously incubated with a buffer } \\
\text { in a time-dependence mode. The } \\
\text { supernatant will be then incubated with } \\
\text { the microbial suspension as well as the } \\
\text { buffer treated materials. }\end{array}$ \\
\hline
\end{tabular}

Table 6 - Table with recommendations for microbiological testing and factors that are to be taken into consideration when choosing the method of assessment. [ADT: Agar diffusion test; DCT: direct contact test MIC: minimum inhibitory concentration; MBC: minimum bactericidal concentration; MBIC: minimum biofilm inhibitory concentration; CFU: colony forming units; CLSM: confocal laser scanning microscopy; EA: enzymatic activity; FISH: fluorescence in situ hybridization; PCR: polymerase chain reaction and nucleic acid methods].



Table 7 - Cell lines suggested for use to test devices for dental applications.

\begin{tabular}{|c|c|c|c|c|c|}
\hline Device & Procedure & Substrate & Tissue & Cell line & ATCC number \\
\hline \multirow{4}{*}{ Implantable } & Oral Surgery & Bone & Bone & MG- 63 & CRL-1427 \\
\hline & Implantology & Bone & Bone & MG- 63 & CRL-1427 \\
\hline & Periapical Surgery & Tooth (Dentin) & Bone & MG- 63 & CRL-1427 \\
\hline & & Oral Mucosa & Fibroblasts & L929 & CCL-1 \\
\hline \multirow{5}{*}{ Non-Implantable } & Restorative & Tooth (Enamel) & $N / A$ & N/A & NA \\
\hline & \multirow[t]{2}{*}{ Dentistry } & Tooth (Dentin-Crown) & $N / A$ & N/A & $N / A$ \\
\hline & & Tooth (Dentin-Root) & $N / A$ & $N / A$ & $N / A$ \\
\hline & \multirow{2}{*}{ Orthodontics } & Oral Mucosa & Fibroblasts & L929 & $\mathrm{CCl}-1$ \\
\hline & & Tooth (Enamel) & $\mathrm{N} / \mathrm{A}$ & N/A & N/A \\
\hline
\end{tabular}

N/A: non-applicable. 


\begin{tabular}{|c|c|c|c|c|c|c|c|c|c|c|c|c|c|c|c|c|}
\hline \multirow{2}{*}{$\begin{array}{l}\text { Material } \\
\text { Type }\end{array}$} & \multicolumn{4}{|c|}{ Metal } & \multicolumn{4}{|c|}{ Polymer } & \multicolumn{4}{|c|}{ Ceramic } & \multicolumn{4}{|c|}{ Natural material } \\
\hline & $\mathrm{B}$ & C & $\mathrm{F}$ & $\mathrm{R}$ & B & C & $\mathrm{F}$ & $\mathrm{R}$ & $\mathrm{B}$ & C & $\mathrm{F}$ & $\mathrm{R}$ & B & C & $\mathrm{F}$ & $\mathrm{R}$ \\
\hline $\mathrm{XRF}$ & $\times$ & $\times$ & $x$ & $x$ & & & & & $x$ & $x$ & $x$ & $x$ & $\times$ & $x$ & $x$ & $x$ \\
\hline NMR & & & & & $\times$ & $\times$ & $\times$ & $x$ & & & & & $\times$ & $x$ & $\times$ & $x$ \\
\hline ICP & $\times$ & $\times$ & $x$ & $x$ & & & & & $x$ & $x$ & $x$ & $\times$ & $\times$ & $x$ & $x$ & $x$ \\
\hline XRD & $\times$ & $\times$ & $x$ & $x$ & & & & & $x$ & $\times$ & $x$ & $x$ & $\times$ & $x$ & $\times$ & $x$ \\
\hline FTIR-ATR & & $\times$ & $x$ & & $x$ & $x$ & $\times$ & $x$ & $x$ & $x$ & $x$ & $x$ & $\times$ & $x$ & $\times$ & $x$ \\
\hline RAMAN & & $x$ & $x$ & & $x$ & $\times$ & $\times$ & $x$ & $\times$ & $x$ & $\times$ & $x$ & $\times$ & $x$ & $x$ & $x$ \\
\hline XPS & & $x$ & $x$ & & & $\times$ & $x$ & & & $x$ & $\times$ & & & $\times$ & $x$ & \\
\hline TOF SIMS & & $x$ & $x$ & & & $\times$ & $x$ & & & $x$ & $x$ & & & $x$ & $x$ & \\
\hline
\end{tabular}

3.3.3.1. Static tests. At least one static test should be performed when evaluating a device used for dental applications. The seeded cells should be assessed by:

- Methylthiazolyldiphenyl-tetrazolium bromide (MTT) assay [13] or other closely related tetrazolium dye-based assays [14] including:

- methoxynitrosulfophenyl-tetrazolium carboxanilide (XTT) assay

o dimethylthiazol-carboxymethoxyphenyl-sulfophenyltetrazolium (MTS) assay

- water-soluble tetrazolium salts (WSTs)

- Scanning electron microscopy (SEM). This method allows visualization of morphological and attachment characteristics of cells grown in direct contact with material specimens

- Live-dead staining and confocal microscopy (CLSM)

- Colony forming unit (CFU) counting after vigorous vortexing and/or sonication (Tween $80^{\circledR}$ might be added for suspending the biofilm cells) [15]

- Cytotoxicity detection kit (lactate dehydrogenase-LDH)

3.3.3.2. Proliferation migration. A proliferation migration test may be performed when evaluating a device used for dental applications. The seeded cells should be assessed by:

- Transwell migration assay

- Cell cycle delay

- Apoptosis us. necrosis

- DNA damage

3.3.3.3. Genotoxicity testing. Two types of genetic toxicology studies are considered important: 1) those measuring direct, irreversible, damage to the DNA that is transmissible to the next cell generation, (i.e., mutagenicity); and 2) those measuring early, potentially reversible effects to DNA or to mechanisms involved in the preservation of the integrity of the genome (genotoxicity). In vitro genotoxicity tests are currently recognized by the Organization for Economic Cooperation and Development, OECD $[16,17]$.

3.3.3.4. Integrated microbial and cellular testing. Using this method, the dental device under study and the controls will be first contaminated by immersion in a microbial inoculum in its corresponding growth medium. The suggested micro- bial strains are listed in Table 3. Next, microbial suspension is removed and samples are washed and cells representative of the studied tissue as defined in Table 7 are suspended in modified culture medium (supplemented with $2 \%$ of the appropriate microbial growth medium) and seeded on the microbe-coated surfaces. All experiments should be performed in triplicate for each type of surface.

\subsubsection{Material characterization methods}

The characterization should be performed on set materials if the material requires mixing and has a setting reaction. The age of testing and any ageing procedures should be specified. The characterization shall include both bulk and surface characterization. If the material is degradable, elution and degradation tests should also be included.

3.3.4.1. Chemical characterization. Chemical characterization needs to be performed in bulk and on the surface. The methods are suggested in Table 8. Furthermore, the nature of the leachate and degraded material also needs to be assessed.

3.3.4.1.1. Bulk characterization. The aim of bulk characterization is the determination of the chemical composition. For organic polymer-based materials characterization by either Fourier transform infrared (FT-IR) spectroscopy in attenuated total reflectance (ATR) mode or Raman spectroscopy should be used. Nuclear magnetic resonance (NMR) spectroscopy can be used both on solid and solubilized materials.

For inorganic materials both elemental and phase analyses need to be carried out. The suggested methods are inductive coupled plasma (ICP) spectroscopy or X-ray fluorescence analysis (XRF) for elemental analyses and FTIR spectroscopy for identification at the molecular level. Crystalline materials may be identified with X-ray diffraction analyses (XRD). The chemical characterization methods are outlined in ISO 10993-18:2009 [18].

3.3.4.1.2. Surface characterization. Surface characterization shall be carried out to identify thin and ultra-thin coatings and their functionalization. The method of choice is X-ray photoelectron spectroscopy (XPS) or time of flight secondary ion mass spectrometry (TOF-SIMS). In some cases, Fourier transform infrared spectroscopy (FT-IR) in the ATR mode or Raman spectroscopy may be used. Surface phase(s) may be analyzed by small angle X-ray scattering (SAXS) or grazing angle X-ray diffraction (GIXD). 
3.3.4.1.3. Characterization of elution/degraded materials. If the device is not inert or it is designed to be absorbed in vivo, the degradation behavior is suggested to be characterized. Degradation and elution are performed according to ISO 10993-9:2009 [19], ISO 10993-13;2010 [20], ISO 10993-14;2009 [21] and ISO 10993-15:2009 [22]. For leachables from organic devices outlined in ISO 10993-13:2010 [20] mass spectroscopy is the suggested method of assessment. For inorganic leachables outlined in ISO 10993-14:2009 [21] and ISO 10993-15:2009 [22] inductively coupled plasma (ICP) spectroscopy is suggested.

If the device is degradable and wear particles are released, the particle size distribution of these particles needs to be assessed. The suggested methods are laser diffraction granulometry, dynamic light scattering or scanning electron microscopy analysis.

3.3.4.2. Physical and mechanical characterization. The physical and mechanical characterization of devices used for dental applications is defined in a number of ISO standards based on the material chemistry and/or specific use. Since the properties of the material surfaces and the bulk materials may be different, additional test methods pertinent to surface characterization are also included together with release assay studies.

3.3.4.2.1. Test methods for implantable materials. Dentistry - membrane materials for guided tissue regeneration in oral and maxillofacial surgery. ISO 22803:2004 [23]

Dentistry - implantable materials for bone filling and augmentation in oral and maxillofacial surgery - contents of a technical file. ISO 22794: 2007 [24].

Dentistry - screw loosening test using cyclic torsional loading for implant body/implant abutment connection of endosseous dental implants. ISO/TR 18130: 2016 [25].

Dentistry - implants. Dynamic loading test for endosseous dental implants. ISO 14801: 2016 [26].

Dentistry - magnetic attachments. ISO 13017: 2012/Amd 1: 2015 [27].

3.3.4.2.2. Test methods for non-implantable materials. Dentistry - zinc oxide/eugenol cements and zinc oxide/noneugenol cements. ISO 3107: 2011 [28]

Dentistry - water-based cements - part 1: powder/liquid acid-base cements. ISO 9917-1: 2007 [29]

Dentistry - water-based cements - part 2: resin-modified cements. ISO 9917-2: 2010 [30].

Dentistry - polymer-based pit and fissure sealants. ISO 6874: 2015 [31].

Dentistry - polymer-based restorative materials. ISO 4049: 2009 [32].

Dentistry — dental amalgam. ISO 24234: 2015 [33].

Dentistry — root canal sealing materials. ISO 6876: 2012 [34]. [35].

Dentistry - root-canal obturating points. ISO 6877: 2006

Dentistry - base polymers - part 1: denture base polymers. ISO 20795-1: 2013 [36].

Dentistry - soft lining materials for removable dentures part 1: materials for short-term use. ISO 10139-1: 2005 [37].
Dentistry - soft lining materials for removable dentures part 2: materials for long-term use. ISO 10139-2: 2016 [38].

Dentistry - metallic materials for fixed and removable restorations and appliances. ISO 22674: 2016 [39].

Dentistry - ceramic materials. ISO 6872: 2015 [40].

Dentistry - polymer based crown and veneering materials. ISO/CD 10477: 2017 [41].

Dentistry - polymer-based luting materials containing adhesive components. ISO/DTS 16506: 2017 [42].

Dentistry - brackets and tubes for use in orthodontics. ISO 27020: 2010 [43].

Dentistry - coiled springs for use in orthodontics. ISO 17254: 2016 [44].

Dentistry — wires for use in orthodontics. ISO 15841: 2014 [45].

Dentistry - base polymers - part 2: orthodontic base polymers. ISO 20795-2:2013 [46].

Dentistry - elastomeric auxiliaries for use in orthodontics. ISO 21606: 2007 [47].

Dentistry - guidance on colour measurement. ISO/TR 28642: 2011 [48].

3.3.4.2.3. Physical characterization of surfaces. Physical surface characterization should be carried out to get details regarding the physical properties, such as structure, roughness and wettability. The methods of choice are surface profilometry, atomic force microscopy (AFM) or scanning electron microscopy (SEM) to obtain information about the homogeneity and roughness of the surface, whilst contact angle measurements will allow the evaluation of the hydrophobicity of the surface (wettability). Physical properties should be analyzed both on freshly prepared surfaces and on aged ones to assess the influence of various factors on the stability and properties of the surface.

3.3.4.2.4. Mechanical testing of surfaces and coatings. The mechanical properties of the surface of a dental device are evaluated by testing the microhardness, scratch testing and adhesion of coatings. The micro-hardness testing for metallic dental devices is described in ISO 6507: 2005 [49]. This test method can be used for other material types ensuring that the number of replicates is higher with resin materials. Nonmetallic material surfaces need to be polished to a high level to enable the accurate measurement of the indentation created by the indenter. If an alternative indenter to the Vickers is used the values can be converted using appropriate tables [50]. Scratch testing is valuable to assess ultra-thin coatings. All the testing is summarized in ISO 1518: 1992 [51]. There are no specific standards to test dental devices for both scratch testing and determination of bond strength of coatings to the device surfaces.

3.3.4.2.5. Release assay studies. The need for pharmaceutical testing of the device will depend on the material properties. Devices used for dental applications may be classified as whether having or not an active component. The former are further sub-classified as releasing or non-releasing systems. In vitro release assays are necessary for the devices releasing active components. The release assays will depend on the chemistry of the components released. If the device is being aged, the artificial ageing method should be specified. Several factors affect the release assay. These factors include: 
- The medium

- Time span

- Temperature

- Static us. dynamic test (indicate type and frequency)

- Condition of device (whether the device is subject to compressive or tensile stress)

- Sample shape

- Volume of solution us. sample surface area ratio

- Cumulative testing us. batch testing

- Solubility of the active component released in the medium (SINK conditions recommended)

These factors should be noted in the report.

The assays performed depend on the nature of the component being released. Organic components should be assessed by High Performance Liquid Chromatography (HPLC) or Ultraviolet (UV) spectrometry. Inorganic components should be assessed by inductively coupled plasma (ICP) spectroscopy. Testing of specific drugs should be performed based on the European Pharmacopoeia [52]. Testing for peptides should be performed using either peptide competition assay (PCA) or colorimetric peptide assay. Validation of the analytical methods should be written in the report. For active releasing compounds, neutralization of the antimicrobial agent must be undertaken prior to testing [53].

\subsubsection{Statistical considerations}

Statistical analysis may vary taking into consideration the following:

- Nature of the material: manually mixed and prepared specimens will need a higher sample number than machined specimens;

- Sample size: appropriate statistical analyses will have to take into consideration the sample size;

- Qualitative us. quantitative analyses. For all analyses, the sample should be homogenous and representative of the material;

- The specimen size should be appropriate to the specific use and the test performed as it affects the statistical analyses.

At least a minimum number of three specimens should be tested. Depending on the test performed, at least three independent measurements should be done per specimen and three replicates should be considered. The replicate testing should be performed by the same operator in the same conditions. For details of statistical analyses refer to ISO 2602:1980 [54]. Level of significance of statistical tests should be set at $5 \%$ or inferior ( $p$ value $\leq 0.05$ ).

\section{Conclusions}

This document outlines the recommendations for characterization and standardization of antimicrobial testing of dental devices. The methods suggested are established methods that have been thoroughly tried and tested and are thus reliable. The full testing methodology is shown in Fig. 2.

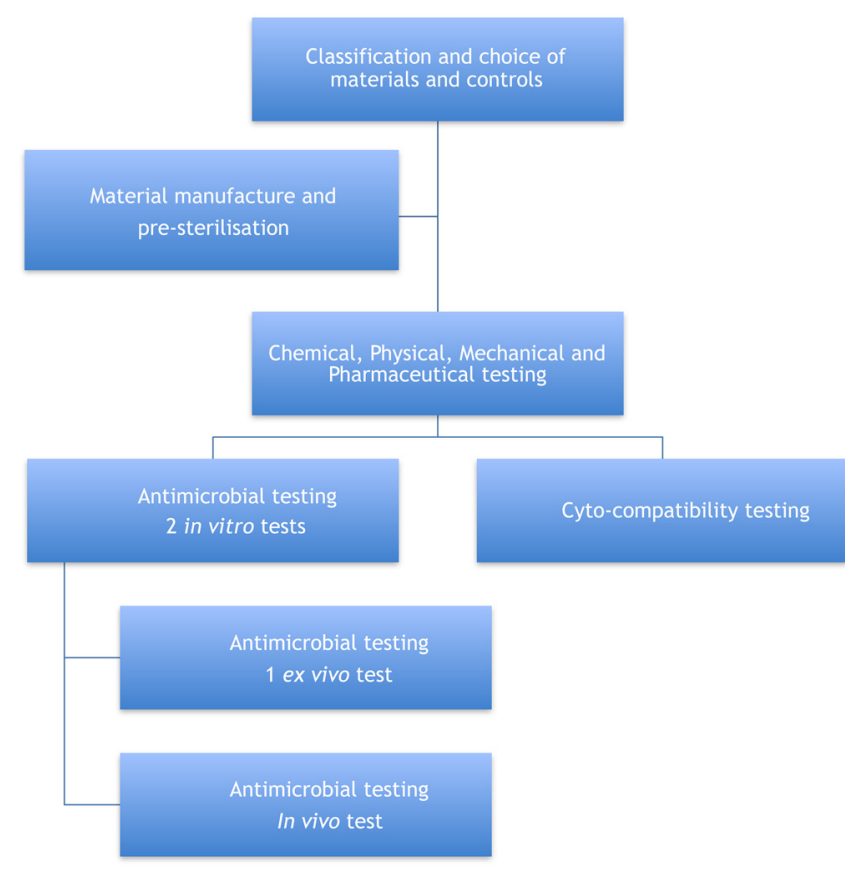

Fig. 2 - Testing methodology proposed.

\section{Acknowledgements}

COST action TD 1305: IPROMEDAI- Improved Protection of Medical Devices Against Infection.

The following are also acknowledged for their contribution in the discussions:

Reto Luginbuehl, University of Bern, Bern, Switzerland; Moter Annette, Charité-Universitätsmedizin Berlin, Germany; Thevissen Karin, KU, Leuven, Belgium; Furtos Gabriel, Raluca Ripan Institute of Research in Chemistry, Babes-Bolyai, University, Romania; Gameiro Paula, Faculdade de Ciências da Universidade do Porto, Portugal; Katsikogianni Maria, Richmond Building. Department of Chemistry and Biosciences. RKT Centre, Advanced Materials Engineering, University of Bradford, UK; Locs Janis, Rudolfs Cimdins Riga, Biomaterials Innovations and Development Centre, Riga Technical University, Riga, Latvia; Miskovic Stankovic Vesna, Department of Physical Chemistry and Electrochemistry, University of Belgrade, Faculty of Technology and Metallurgy, Belgrade; Chakraborti Soumyananda, Malopolska Centre of Biotechnology Krakow, Poland; Çimenoğlu Huseyin, Istanbul Technical University, Istanbul, Turkey; Farzaneh Moghtader, Zanjan University of Medical Sciences, Turkey; Albu Kaya Amadalina, INCDTP - Division ICPI, Collagen Department, Bucharest, Romania; Pavilan Cornelia, University of Basel, Switzerland; Bakopoulou Athina, Department of Prosthodontics, School of Dentistry, Aristotle University of Thessaloniki, Greece, Bousnaki Maria, Department of Prosthodontics, School of Dentistry, Aristotle University of Thessaloniki, Greece.

\section{REFERENCES}

[1] REGULATION (EU) 2017/745. https://eur-lex.europa.eu/ legal-content/EN/TXT/PDF/?uri=CELEX:32017R0745. 
[2] International Standards Organization, ISO/TS 11405 Dental materials - testing of adhesion to tooth structure; 2015.

[3] International Standards Organization, ISO 11135 Sterilization of health-care products - ethylene oxide requirements for the development, validation and routine control of a sterilization process for medical devices; 2014

[4] International Standards Organization, ISO 11137-1 Sterilization of health care products - radiation - part 1: requirements for development, validation and routine control of a sterilization process for medical devices; 2006.

[5] International Standards Organization, ISO 17665-1 Sterilization of health care products - moist heat - part 1: requirements for the development, validation and routine control of a sterilization process for medical devices; 2006.

[6] International Standards Organization, ISO 20857 Sterilization of health care products - dry heat requirements for the development, validation and routine control of a sterilization process for medical devices; 2010.

[7] International Standards Organization, ISO 25424 Sterilization of medical devices - low temperature steam and formaldehyde - requirements for development, validation and routine control of a sterilization process for medical devices; 2009.

[8] International Standards Organization, ISO 14160 Sterilization of health care products - liquid chemical sterilizing agents for single-use medical devices utilizing animal tissues and their derivatives - requirements for characterization, development, validation and routine control of a sterilization process for medical devices; 2011.

[9] International Standards Organization, ISO 11737-1 Sterilization of medical devices - microbiological methods - part 1: determination of a population of microorganisms on products; 2006.

[10] International Standards Organization, ISO 11737-2 Sterilization of medical devices - microbiological methods - part 2: tests of sterility performed in the definition, validation and maintenance of a sterilization process; 2009.

[11] International Standards Organization, ISO 7405 Dentistry evaluation of biocompatibility of medical devices used in dentistry; 2008.

[12] International Standards Organization, ISO 10993-5 Biological evaluation of medical devices part 5: tests for in vitro cytotoxicity; 2009.

[13] Mosmann T. Rapid calorimetric assay for cellular growth and survival; application to proliferation and cytotoxicity assays. J Immunol Method 1983;65:55-63.

[14] Berridge MV, Herst PM, Tan AS. Tetrazolium dyes as tools in cell biology: new insights into their cellular reduction. Biotechnol Annu Rev 2005;11:127-52.

[15] Polyethyleneimine nanoparticles incorporated into resin composite cause cell death and trigger biofilm stress in vivo. Proc Natl Acad Sci U S A 2010;107(51):22038-43.

[16] https://www.oecd.org/chemicalsafety/testing/Genetic \%20Toxicology\%20Guidance\%20Document\%20Aug \%2031\%202015.pdf.

[17] Cimino MC. Comparative overview of current international strategies and guidelines for genetic toxicology testing for regulatory purposes. Environ Mol Mutagen 2006;47:362-90. Erratum, Environ. and Molec. Mutagenesis 2006;47:746.

[18] International Standards Organization, ISO 10993-18 Biological evaluation of medical devices - part 18: chemical characterization of materials; 2005.

[19] International Standards Organization, ISO 10993-9 Biological evaluation of medical devices - part. 9: framework for identification and quantification of potential degradation products; 2016.

[20] International Standards Organization, ISO 10993-13 Biological evaluation of medical devices - part. 13: identification and quantification of degradation products from polymeric medical devices; 2010.

[21] International Standards Organization, ISO 10993-14 Biological evaluation of medical devices - part. 14: identification and quantification of degradation products from ceramics; 2001.

[22] International Standards Organization, ISO 10993-15 Biological evaluation of medical devices - part. 15: identification and quantification of degradation products from metals and alloys; 2000.

[23] International Standards Organization, ISO 22803 Dentistry - membrane materials for guided tissue regeneration in oral and maxillofacial surgery; 2004.

[24] International Standards Organization, ISO 22794 Dentistry - implantable materials for bone filling and augmentation in oral and maxillofacial surgery - contents of a technical file; 2007

[25] International Standards Organization, ISO/TR 18130 Dentistry - screw loosening test using cyclic torsional loading for implant body/implant abutment connection of endosseous dental implants; 2016.

[26] International Standards Organization, ISO 14801 Dentistry - implants-dynamic loading test for endosseous dental implants; 2016.

[27] International Standards Organization, ISO 13017 Dentistry — magnetic attachments; 2012.

[28] International Standards Organization, ISO 3107 Dentistry zinc oxide/eugenol cements and zinc oxide/non-eugenol cements; 2011.

[29] International Standards Organization, ISO 9917-1 Dentistry - water-based cements - part 1: powder/liquid acid-base cements; 2007.

[30] International Standards Organization, ISO 9917-2 Dentistry - water-based cements - part 2: resin-modified cements; 2010.

[31] International Standards Organization, ISO 6874 Dentistry polymer-based pit and fissure sealants; 2015

[32] International Standards Organization, ISO 4049 Dentistry polymer-based restorative materials; 2009.

[33] International Standards Organization, ISO 24234 Dentistry - dental amalgam; 2015.

[34] International Standards Organization, ISO 6876 Dentistry root canal sealing materials; 2012

[35] International Standards Organization, ISO 6877 Dentistry root-canal obturating points; 2006.

[36] International Standards Organization, ISO 20795-1 Dentistry — base polymers - part 1: denture base polymers; 2013.

[37] International Standards Organization, ISO 10139-1 Dentistry — soft lining materials for removable dentures - part 1 : materials for short-term use; 2005.

[38] International Standards Organization, ISO 10139-2 Dentistry - soft lining materials for removable dentures - part 2: materials for long-term use; 2016.

[39] International Standards Organization, ISO 22674 Dentistry - metallic materials for fixed and removable restorations and appliances; 2016.

[40] International Standards Organization, ISO 6872 Dentistry ceramic materials; 2015.

[41] International Standards Organization, ISO/CD 10477 Dentistry - polymer based crown and veneering materials; 2018.

[42] International Standards Organization, ISO/TS 16506 Dentistry - polymer-based luting materials containing adhesive components; 2017.

[43] International Standards Organization, ISO 27020 Dentistry — brackets and tubes for use in orthodontics; 2010.

[44] International Standards Organization, ISO 17254 Dentistry — coiled springs for use in orthodontics; 2016. 
[45] International Standards Organization, ISO 15841 Dentistry — wires for use in orthodontics; 2014.

[46] International Standards Organization, ISO 20795-2 Dentistry - base polymers - part 2: orthodontic base polymers; 2013.

[47] International Standards Organization, ISO 21606 Dentistry - elastomeric auxiliaries for use in orthodontics; 2007.

[48] International Standards Organization, ISO/TR 28642 Dentistry — guidance on colour measurement; 2011.

[49] International Standards Organization, ISO 6507-1 Metallic materials - Vickers hardness test - part 1: test method; 2005.

[50] International Standards Organization, ISO 6507-4 Metallic materials - Vickers hardness test - part 4: tables of hardness values; 2005.
[51] International Standards Organization, ISO 1518 Paints and varnishes - scratch test; 1992.

[52] European Pharmacopoeia. $9^{\text {th }}$ Edition.

[53] International Standards Organization, BS EN 1040 Chemical disinfectants and antiseptics. Quantitative suspension test for the evaluation of basic bactericidal activity of chemical disinfectants and antiseptics. Test method and requirements (phase 1); 2005.

[54] International Standards Organization, ISO 2602 Statistical interpretation of test results; 1980. 